\title{
Superconvergence of a finite element method for the time-fractional diffusion equation with a time-space dependent diffusivity
}

$\mathrm{Na} \mathrm{An}^{1 *}$

\section{"Correspondence:}

annabetter@sdnu.edu.cn

${ }^{1}$ School of Mathematics and

Statistics, Shandong Normal

University, 250014, Jinan, China

\section{Springer}

\begin{abstract}
In this work, a time-fractional diffusion problem with a time-space dependent diffusivity is considered. The solution of such a problem has a weak singularity at the initial time $t=0$. Based on the L1 scheme in time on a graded mesh and the conforming finite element method in space on a uniform mesh, the fully discrete L1 conforming finite element method (L1 FEM) of a time-fractional diffusion problem is investigated. The error analysis is based on a nonstandard discrete Gronwall inequality. The final superconvergence result shows that an optimal grading of the temporal mesh should be selected as $r \geq(2-\alpha) / \alpha$. Numerical results confirm that our analysis is sharp.
\end{abstract}

Keywords: Time-fractional diffusion; Caputo derivative; Finite element method; Superconvergence

\section{Introduction}

During the past few decades, several physical models have been developed in the form of fractional differential equations. They can be used to modeling certain phenomena in fractal networks, signal processing, turbulent flows, wave propagation, etc. Compared with a classical integral-order equation, the main advantage of the fractional order equation is that it provides an excellent instrument for the description of memory and hereditary properties of various physical models.

In this paper, we consider the following time-fractional initial-boundary value problems (IBVPs):

$$
\begin{aligned}
& D_{t}^{\alpha} u-\nabla \cdot(a(x, t) \nabla u)=f(x, t) \quad \text { on } Q:=\Omega \times(0, T] \\
& u(x, 0)=u_{0}(x) \quad \text { for } x \in \bar{\Omega}
\end{aligned}
$$

with $\left.u\right|_{\partial \Omega}=0$ for $t \in[0, T]$. Here we assume that the spatial domain $\Omega \subset \mathbb{R}^{2}$ is a convex polyhedral domain. We assume that $u_{0} \in C(\bar{\Omega}), f \in C(\bar{Q})$, and the diffusivity coefficient

(c) The Author(s) 2020. This article is licensed under a Creative Commons Attribution 4.0 International License, which permits use, sharing, adaptation, distribution and reproduction in any medium or format, as long as you give appropriate credit to the original author(s) and the source, provide a link to the Creative Commons licence, and indicate if changes were made. The images or other third party material in this article are included in the article's Creative Commons licence, unless indicated otherwise in a credit line to the material. If material is not included in the article's Creative Commons licence and your intended use is not permitted by statutory regulation or exceeds the permitted use, you will need to obtain permission directly from the copyright holder. To view a copy of this licence, visit http://creativecommons.org/licenses/by/4.0/. 
$a(x, t)$ satisfies

$$
\lambda^{-1} \leq a(x, t) \leq \lambda \quad \text { for a fixed constant } \lambda \geq 1 .
$$

In (1a), $D_{t}^{\alpha}$ is the Caputo fractional derivative operator defined by

$$
D_{t}^{\alpha} v(x, t)=\frac{1}{\Gamma(1-\alpha)} \int_{0}^{t}(t-s)^{-\alpha} \frac{\partial v(x, s)}{\partial s} d s .
$$

The time-fractional diffusion equation (1a)-(1b) has been proved to be a very valuable tool in modeling complex systems, for example, charge carrier transport in amorphous semiconductors [8], nuclear magnetic resonance (NMR) diffusometry in percolative structures [18], rouse or reptation dynamics in polymeric systems [40], transport on fractal geometries [33], etc. The analytical solution of some fractional partial differential equations can be obtained by Laplace transform, differential transform method, and fractional complex transform, etc. [1, 25, 28-30]. But most of the equations have no analytical solution, so it is very important to solve them numerically.

Numerical methods for time-fractional IBVPs with constant or time-independent diffusion parameter have received a huge amount of attention over the last decade. For such problems, several numerical methods have been proposed and analyzed, such as finite difference method [7, 19-21, 27, 36, 38], finite element method [6, 32, 39, 41, 43, 44, 48], discontinuous Galerkin (DG) methods [3, 4, 9-11,31, 34], spectral method [23], and finite volume method $[15,46]$, etc. The time-fractional IBVPs (1a)-(1b) with time-space dependent diffusivity is indeed very interesting and also practically important, and the numerical solutions of this problems were considered by a few authors only. Alikhanov [2] constructed an L2-1 $\sigma$ scheme for problem (1a) $-(1 \mathrm{~b})$, and the error analysis of this scheme was based on the sufficient smoothness assumption of the solution. Mustapha [17] studied a semidiscrete Galerkin finite element method for time-fractional diffusion equations with time-space dependent diffusivity, and the optimal error bounds in spatial $L^{2}$ - and $H^{1}$-norms were derived for smooth and nonsmooth initial data by using novel energy arguments. The regularity result about the solutions of the subdiffusion model was proved for both nonsmooth initial data and incompatible source term by Jin [16], and a complete error analysis was presented for a fully discrete conforming FEM. Zhang and Shi [45] proposed a fully discrete L1 mixed finite element method for time fractional diffusion equation with a smooth solution, and a novel result of the consistency error estimate with order $O\left(h^{2}\right)$ of the bilinear element was obtained. Zhao et al. [47] presented a fully discrete L1 finite element method for multiterm time fractional diffusion equation with constant diffusivity, and a superconvergence result for $H^{1}$-norm estimate was obtained. Yin et al. [42] presented two families of novel fractional $\theta$-methods to solve the fractional cable model, and an optimal convergence result with $\mathrm{O}\left(\tau^{2}+h^{k+1}\right)$ for smooth solutions was obtained. Syed et al. [26] proposed a homotopy analysis method for the space-time fractional Korteweg-de Vries (KdV) equation. Huang and Stynes [13] proposed a fully discrete finite element method for the multiterm time fractional diffusion equation with a weak singularity solution, and a simple postprocessing of the computed solution yielded a higher order of convergence in the spatial direction.

Imitating [16, Sect. 2], we derive that the solution of (1a)-(1b) satisfies

$$
\|u(\cdot, t)\|_{2} \leq C, \quad\left\|\partial_{t}^{l} u(\cdot, t)\right\|_{2} \leq C\left(1+t^{\alpha-l}\right), \quad\left\|D_{t}^{\alpha} u(\cdot, t)\right\|_{2} \leq C
$$


for $l=0,1,2$ and $0<t \leq T$. The aim of this paper is constructing a fully discrete conforming finite element method for time-fractional IBVPs (1a)-(1b) with a weak singularity solution (4), and then the superconvergence result in $H^{1}$-norm of this method will be analyzed.

The paper is structured as follows. In Sect. 2, several operators are introduced. In Sect. 3 the L1 discretization on a graded temporal mesh of the Caputo temporal derivative is presented, and then the finite element discretization of the spatial component of the differential operator is described. In Sect. 4 an optimal $H^{1}(\Omega)$ convergence bound for the computed solution is derived, and a simple postprocessing of the computed solution will yield a higher order of convergence in the spatial direction. Finally, numerical results in Sect. 5 show that our theoretical results are optimal.

Notation. $C$ and $K$ are generic constants that are independent of the mesh parameters $N$ and $h$. We write $\|\cdot\|$ for the norm in $L^{2}(\Omega)$. For each $q \in \mathbb{N}$, the notation $H^{q}(\Omega)$ is used for the standard Sobolev space with its associated norm $\|\cdot\|_{q}$ and seminorm $|\cdot|_{q}$.

\section{Preliminaries}

Let $\mathcal{T}_{h}$ be a quasiuniform partition of $\Omega$ into element $K_{m}$ for $m=1, \ldots, M$, and $h=$ $\max _{1 \leq m \leq M}\left\{\operatorname{diam}\left(K_{m}\right)\right\}$ be the mesh size. Then we define the following bilinear finite element spaces:

$$
V_{h}:=\left\{v_{h} \in H_{0}^{1}(\Omega):\left.v_{h}\right|_{K_{m}} \in \operatorname{span}\{1, x, y, x y\} \text { for } m=1,2, \ldots, M\right\}
$$

and

$$
V_{0 h}:=\left\{v_{h} \in V_{h}:\left.v_{h}\right|_{\partial \Omega}=0\right\}
$$

Next, we will introduce three operators, which are used in finite element analyses of timedependent problems [37]. First, we define the $L^{2}$ projector $P_{h}: L^{2}(\Omega) \rightarrow V_{0 h}$ by $\left(P_{h} w, v_{h}\right)=$ $\left(w, v_{h}\right) \forall v_{h} \in V_{0 h}$. By [5, (1.2)], one has

$$
\left\|\nabla P_{h} v\right\| \leq K\|\nabla v\| \quad \text { for all } v \in H_{0}^{1}(\Omega) .
$$

Next we need a time-dependent Ritz projector $R_{h}(t): H_{0}^{1}(\Omega) \rightarrow V_{0 h}$ defined by $(a(\cdot, t) \times$ $\left.\nabla R_{h}(t) w, \nabla v_{h}\right)=\left(a(\cdot, t) \nabla w, \nabla v_{h}\right) \forall v_{h} \in V_{0 h}$. For a fixed $k \geq 0$, since $V_{0 h} \subset H_{0}^{1}(\Omega)$ is the space of piecewise polynomials of degree at most $k$, it is well known $[24,(3.2)]$ that

$$
\left\|w-R_{h}(t) w\right\|+h\left\|w-R_{h}(t) w\right\|_{1} \leq C h^{k+1}|w|_{k+1} \quad \forall w \in H^{k+1}(\Omega) \cap H_{0}^{1}(\Omega) .
$$

In order to obtain our optimal $H^{1}$-norm convergence and superconvergence results given in Sect. 4, we introduce a time-dependent discrete Laplacian $\Delta_{h}(t): V_{0 h} \rightarrow V_{0 h}$ defined by

$$
\left(\Delta_{h}(t) v, w\right)=-(a(\cdot, t) \nabla v, \nabla w) \quad \forall v, w \in V_{0 h}
$$

which will be used to convert the integral form L1 FEM (16) to the differential form scheme (17). According to [16, p. 12], we have that $\Delta_{h}(t): V_{0 h} \rightarrow V_{0 h}$ is bounded and invertible on 
$V_{0 h}$ under condition (2). Imitating [37, p. 11], one has

$$
\begin{aligned}
\left(\Delta_{h}(t) R_{h}(t) v, \chi\right) & =-\left(a(\cdot, t) \nabla R_{h}(t) v, \nabla \chi\right)=-(a(\cdot, t) \nabla v, \nabla \chi) \\
& =(\nabla \cdot(a(\cdot, t) \nabla v), \chi) \\
& =\left(P_{h} \nabla \cdot(a(\cdot, t) \nabla v), \chi\right), \quad \forall \chi \in V_{0 h} .
\end{aligned}
$$

Thus these three operators are related by

$$
\Delta_{h}(t) R_{h}(t) v=P_{h} \nabla \cdot(a(\cdot, t) \nabla v), \quad \forall v \in H^{2}(\Omega) .
$$

\section{Temporal graded meshes; the L1 FEM}

In this section, the well-known L1 scheme on graded meshes will be introduced, and then we present a fully discrete conforming finite element method.

Let $N$ be a positive integer. Set $t_{n}=T(n / N)^{r}$ for $n=0,1, \ldots, N$, where the mesh grading constant $r \geq 1$ is chosen by the user. Set $\tau_{n}=t_{n}-t_{n-1}$ for $n=0,1, \ldots, N$.

For $n \geq 1$, the Caputo fractional derivative $D_{t}^{\alpha} u\left(x, t_{n}\right)$ of (3) can be approximated by the well-known L1 formula:

$$
D_{t}^{\alpha} u\left(x, t_{n}\right) \approx D_{N}^{\alpha} u^{n}:=\frac{d_{n, 1}}{\Gamma(2-\alpha)} u^{n}-\frac{d_{n, n}}{\Gamma(2-\alpha)} u^{0}+\frac{1}{\Gamma(2-\alpha)} \sum_{i=1}^{n-1} u^{n-i}\left(d_{n, i+1}-d_{n, i}\right),
$$

where $d_{n, i}:=\left[\left(t_{n}-t_{n-i}\right)^{1-\alpha}-\left(t_{n}-t_{n-i+1}\right)^{1-\alpha}\right] / \tau_{n-i+1}$ for $i=1, \ldots, n$. Note that $d_{n, 1}=\tau_{n}^{-\alpha}$. It is easily to see that

$$
d_{n, i+1}<d_{n, i} \text { for } 0 \leq i \leq n-1 \leq N-1 .
$$

Imitating [36, Lemma 5.2], we derive the following truncation error of the L1 scheme (9).

Lemma 3.1 Assume the solution of (1a)-(1b) satisfies (4). For all $\left(x, t_{n}\right) \in Q$, one has

$$
\left\|D_{t}^{\alpha} u\left(x, t_{n}\right)-D_{N}^{\alpha} u\left(x, t_{n}\right)\right\|_{1} \leq C n^{-\min \{2-\alpha, r \alpha\}} .
$$

As in [36, (4.6)], define the positive real numbers $\theta_{n, j}$, for $n=1,2, \ldots, N$ and $j=1,2, \ldots, n-$ 1 , by

$$
\theta_{n, n}=1, \quad \theta_{n, j}=\sum_{k=1}^{n-j} \tau_{n-k}^{\alpha}\left(d_{n, k}-d_{n, k+1}\right) \theta_{n-k, j} .
$$

Observe that (10) implies $\theta_{n, j}>0$ for all $n, j$. Furthermore, as in [36, Lemma 4.3], for $n=$ $1,2, \ldots, N$, one has

$$
\tau_{n}^{\alpha} \sum_{j=1}^{n} j^{-\beta} \theta_{n, j} \leq \frac{T^{\alpha} N^{-\beta}}{1-\alpha}, \quad \text { provided that } \beta \leq r \alpha .
$$

Next, we will state a nonstandard Gronwall inequality, which is given in [12, Lemma 4.4]. 
Lemma 3.2 Assume that sequences $\left\{\xi^{n}\right\}_{n=1}^{\infty},\left\{\eta^{n}\right\}_{n=1}^{\infty}$ are nonnegative and the grid function $\left\{v^{n}: n=0,1, \ldots, N\right\}$ satisfies $v^{0} \geq 0$ and

$$
\left(D_{N}^{\alpha} v^{n}\right) v^{n} \leq \xi^{n} v^{n}+\left(\eta^{n}\right)^{2} \text { for } n=1,2, \ldots, N
$$

Then

$$
v^{n} \leq v^{0}+\tau_{n}^{\alpha} \Gamma(2-\alpha) \sum_{j=1}^{n} \theta_{n, j} \xi^{j}+\sqrt{T^{\alpha} \Gamma(1-\alpha)} \max _{1 \leq j \leq n} \eta^{j} \quad \text { for } n=1,2, \ldots, N,
$$

where $\theta_{n, j}$ is defined by (11).

Imitating the proof of [14, Lemma 4.2], we derive the following property of the L1 scheme, which will be used in our later analysis.

Lemma 3.3 Let the functions $v^{j}=v\left(\cdot, t^{j}\right)$ be in $L^{2}(\Omega)$ for $j=0,1, \ldots, N$. Then the discrete $L 1$ scheme satisfies

$$
\left(a\left(\cdot, t_{n}\right) D_{N}^{\alpha} v^{n}, v^{n}\right) \geq\left(D_{N}^{\alpha}\left\|\sqrt{a\left(\cdot, t_{n}\right)} v^{n}\right\|\right)\left\|\sqrt{a\left(\cdot, t_{n}\right)} v^{n}\right\| \quad \text { for } n=1,2, \ldots, N .
$$

Proof Let $n \in\{1,2, \ldots, N\}$. Applying $a(x, t)>0$ and Cauchy-Schwarz inequality, one has

$$
\begin{aligned}
\left(a\left(\cdot, t_{n}\right) D_{N}^{\alpha} v^{n}, v^{n}\right)= & \frac{d_{n, 1}}{\Gamma(2-\alpha)}\left(a\left(\cdot, t_{n}\right) v^{n}, v^{n}\right)-\frac{d_{n, n}}{\Gamma(2-\alpha)}\left(a\left(\cdot, t_{n}\right) v^{0}, v^{n}\right) \\
& -\frac{1}{\Gamma(2-\alpha)} \sum_{i=1}^{n-1}\left(d_{n, i}-d_{n, i+1}\right)\left(a\left(\cdot, t_{n}\right) v^{n-i}, v^{n}\right) \\
\geq & \frac{d_{n, 1}}{\Gamma(2-\alpha)}\left\|\sqrt{a\left(\cdot, t_{n}\right)} v^{n}\right\|^{2}-\frac{d_{n, n}}{\Gamma(2-\alpha)}\left\|\sqrt{a\left(\cdot, t_{n}\right)} v^{0}\right\|\left\|\sqrt{a\left(\cdot, t_{n}\right)} v^{n}\right\| \\
& -\frac{1}{\Gamma(2-\alpha)} \sum_{i=1}^{n-1}\left(d_{n, i}-d_{n, i+1}\right)\left\|\sqrt{a\left(\cdot, t_{n}\right)} v^{n-i}\right\|\left\|\sqrt{a\left(\cdot, t_{n}\right)} v^{n}\right\| \\
= & \left(D_{N}^{\alpha}\left\|\sqrt{a\left(\cdot, t_{n}\right)} v^{n}\right\|\right)\left\|\sqrt{a\left(\cdot, t_{n}\right)} v^{n}\right\|,
\end{aligned}
$$

where we used $d_{n, i}>d_{n, i+1}>0$.

\subsection{The L1 FEM}

To begin, our problem (1a)-(1b) will be discretized only in space applying a conforming finite element method. Then the semidiscrete FEM reads: seek $u_{h}(\cdot, t) \in V_{0 h}$ for each $t \in$ $(0, T]$ such that

$$
\left(D_{t}^{\alpha} u_{h}, v_{h}\right)+\left(a(x, t) \nabla u_{h}, \nabla v_{h}\right)=\left(f, v_{h}\right) \quad \text { with } u_{h}^{0}=R_{h}\left(t_{0}\right) u_{0} \text { and all } v_{h} \in V_{0 h}
$$

Applying the L1 scheme (9) to discretize (15) in the temporal domain, the fully discrete L1 FEM is: seek $u_{h}^{n} \in V_{0 h}$ such that

$$
\left(D_{N}^{\alpha} u_{h}^{n}, v_{h}\right)+\left(a\left(x, t_{n}\right) \nabla u_{h}^{n}, \nabla v_{h}\right)=\left(f^{n}, v_{h}\right) \quad \text { for } n=1, \ldots, N \text { and all } v_{h} \in V_{0 h} \text {. }
$$


Invoking (7), the L1 FEM (16) takes the form: find $u_{h}^{n} \in V_{0 h}$ for $n=0,1, \ldots, N$ such that

$$
\left(D_{N}^{\alpha} u_{h}^{n}, v_{h}\right)-\left(\Delta_{h}\left(t_{n}\right) u_{h}^{n}, v_{h}\right)=\left(P_{h} f^{n}, v_{h}\right) \quad \text { for } n=1, \ldots, N \text { and all } v_{h} \in V_{0 h}
$$

with $u_{h}^{0}=R_{h}\left(t_{0}\right) u_{0}$. This formulation of our L1 FEM can be written as: find $u_{h}^{n} \in V_{0 h}$ for $n=0,1, \ldots, N$ such that

$$
D_{N}^{\alpha} u_{h}^{n}-\Delta_{h}\left(t_{n}\right) u_{h}^{n}=P_{h} f^{n} \quad \text { with } u_{h}^{0}=R_{h}\left(t_{0}\right) u_{0} \text { and } n=1, \ldots, N \text {, }
$$

where $D_{N}^{\alpha} u_{h}^{n}, \Delta_{h}\left(t_{n}\right) u_{h}^{n}$ and $P_{h} f^{n}$ all lie in $V_{0 h}$ are used.

\section{Superconvergence of the L1 FEM}

In this section, a superconvergence bound for $\left\|\nabla R_{h} u^{n}-\nabla u_{h}^{n}\right\|$ will be presented, and then a superconvergence result of the L1 FEM (17) will be derived.

Let $u^{n}$ and $u_{h}^{n}$ be the solutions of (1a)-(1b) and (16), respectively, at time $t=t_{n}$ for $n=$ $0,1, \ldots, N$. In order to facilitate the error analysis, denote $\zeta^{n}:=R_{h}\left(t_{n}\right) u^{n}-u_{h}^{n}$ and $\rho^{n}:=$ $R_{h}\left(t_{n}\right) u^{n}-u^{n}$. Then we write

$$
u^{n}-u_{h}^{n}=\left(R_{h}\left(t_{n}\right) u^{n}-u_{h}^{n}\right)-\left(R_{h}\left(t_{n}\right) u^{n}-u^{n}\right)=\zeta^{n}-\rho^{n},
$$

The error of $\rho^{n}$ can be approximated immediately applying (6), but the approximation of $\zeta^{n}$ is difficult, and we estimate it now. From (1a), (8), and (17), one has

$$
\begin{aligned}
D_{N}^{\alpha} \zeta^{n}-\Delta_{h}\left(t_{n}\right) \zeta^{n} & =\left[R_{h}\left(t_{n}\right) D_{N}^{\alpha} u^{n}-\Delta_{h}\left(t_{n}\right) R_{h}\left(t_{n}\right) u^{n}\right]-\left(D_{N}^{\alpha} u_{h}^{n}-\Delta_{h}\left(t_{n}\right) u_{h}^{n}\right) \\
& =\left(R_{h}\left(t_{n}\right)-P_{h}\right) D_{N}^{\alpha} u^{n}+P_{h}\left(D_{N}^{\alpha} u^{n}-\nabla \cdot\left(a\left(\cdot, t_{n}\right) \nabla u^{n}\right)\right)-P_{h} f^{n} \\
& =P_{h}\left(R_{h}\left(t_{n}\right)-I\right) D_{N}^{\alpha} u^{n}+P_{h}\left(f^{n}-\varphi^{n}\right)-P_{h} f^{n} \\
& =P_{h}\left(D_{N}^{\alpha} \rho^{n}-\varphi^{n}\right),
\end{aligned}
$$

where $\varphi^{n}:=D_{t}^{\alpha} u\left(x, t_{n}\right)-D_{N}^{\alpha} u\left(x, t_{n}\right)$.

Now the optimal-rate convergence of our method in $L^{\infty}\left(H^{1}\right)$ and a superconvergence bound for $\left\|\nabla R_{h} u^{n}-\nabla u_{h}^{n}\right\|$ will be stated in the following theorem.

Theorem 4.1 (Error estimate for the L1 FEM) Assume $\|u\|_{L^{\infty}\left(H^{2}\right)}$ and $\left\|D_{t}^{\alpha} u\right\|_{L^{\infty}\left(H^{2}\right)}$ are finite. Let $u^{n}$ and $u_{h}^{n}$ be the solutions of (1a)-(1b) and (16), respectively. Then for $n=$ $1,2, \ldots, N$, there exists a constant $C$ such that

$$
\begin{aligned}
& \left\|\nabla u^{n}-\nabla u_{h}^{n}\right\| \leq C\left(h+N^{-\min \{2-\alpha, r \alpha\}}\right), \\
& \left\|\nabla R_{h}\left(t_{n}\right) u^{n}-\nabla u_{h}^{n}\right\| \leq C\left(h^{2}+N^{-\min \{2-\alpha, r \alpha\}}\right) .
\end{aligned}
$$

Proof Fix $n \in\{1,2, \ldots, N\}$. Multiplying (19) by $-\Delta_{h}\left(t_{n}\right) \zeta^{n}$ and integrating over $\Omega$, one has

$$
-\left(D_{N}^{\alpha} \zeta^{n}, \Delta_{h}\left(t_{n}\right) \zeta^{n}\right)+\left\|\Delta_{h}\left(t_{n}\right) \zeta^{n}\right\|^{2}=-\left(P_{h}\left(D_{N}^{\alpha} \rho^{n}-\varphi^{n}\right), \Delta_{h}\left(t_{n}\right) \zeta^{n}\right) .
$$

It is obvious that

$$
D_{N}^{\alpha} \rho^{n}=D_{N}^{\alpha} \rho^{n}-D_{t}^{\alpha} \rho^{n}+D_{t}^{\alpha} \rho^{n}=\left(D_{t}^{\alpha} u^{n}-D_{N}^{\alpha} u^{n}\right)-R_{h}\left(t_{n}\right)\left(D_{t}^{\alpha} u^{n}-D_{N}^{\alpha} u^{n}\right)+D_{t}^{\alpha} \rho^{n}
$$


Inserting (23) into (22) and recalling the definition (7) of $\Delta_{h}\left(t_{n}\right)$ yields

$$
\begin{aligned}
& \left(a\left(\cdot, t_{n}\right) D_{N}^{\alpha}\left(\nabla \zeta^{n}\right), \nabla \zeta^{n}\right)+\left\|\Delta_{h}\left(t_{n}\right) \zeta^{n}\right\|^{2} \\
& \quad=-\left(D_{t}^{\alpha} \rho^{n}, \Delta_{h}\left(t_{n}\right) \zeta^{n}\right)+\left(a\left(\cdot, t_{n}\right) \nabla P_{h}\left(-R_{h}\left(t_{n}\right) \varphi^{n}\right), \nabla \zeta^{n}\right)
\end{aligned}
$$

Applying Lemma 3.3 and Cauchy-Schwarz inequality, one has

$$
\begin{aligned}
& D_{N}^{\alpha}\left\|\sqrt{a\left(\cdot, t_{n}\right)} \nabla \zeta^{n}\right\|\left\|\sqrt{a\left(\cdot, t_{n}\right)} \nabla \zeta^{n}\right\| \\
& \quad \leq \frac{1}{4}\left\|D_{t}^{\alpha} \rho^{n}\right\|^{2}+\left\|\sqrt{a\left(\cdot, t_{n}\right)} \nabla P_{h}\left(R_{h}\left(t_{n}\right) \varphi^{n}\right)\right\|\left\|\sqrt{a\left(\cdot, t_{n}\right)} \nabla \zeta^{n}\right\| .
\end{aligned}
$$

Invoking (5), (6), and (2), we get

$$
D_{N}^{\alpha}\left\|\sqrt{a\left(\cdot, t_{n}\right)} \nabla \zeta^{n}\right\|\left\|\sqrt{a\left(\cdot, t_{n}\right)} \nabla \zeta^{n}\right\| \leq C h^{4}+\sqrt{\lambda} K\left\|\nabla\left(R_{h}\left(t_{n}\right) \varphi^{n}\right)\right\|\left\|\sqrt{a\left(\cdot, t_{n}\right)} \nabla \zeta^{n}\right\| .
$$

Observe that (24) is a particular case of (13). Thus we can invoke Lemma 3.2 to get

$$
\begin{aligned}
\left\|\sqrt{a\left(\cdot, t_{n}\right)} \nabla \zeta^{n}\right\| \leq & \left\|\sqrt{a\left(\cdot, t_{n}\right)} \nabla \zeta^{0}\right\|+\sqrt{\lambda} K \tau_{n}^{\alpha} \Gamma(2-\alpha) \sum_{j=1}^{n} \theta_{n, j}\left\|\nabla\left(R_{h}\left(t_{j}\right) \varphi^{j}\right)\right\| \\
& +\sqrt{T^{\alpha} \Gamma(1-\alpha)} \max _{1 \leq j \leq n} C h^{2} .
\end{aligned}
$$

Inequality $\left\|\nabla R_{h}(t) w\right\| \leq \lambda\|\nabla w\| \forall w \in H_{0}^{1}(\Omega)$ follows easily from the definition of $R_{h}(t)$. Hence

$$
\begin{aligned}
& \left\|\sqrt{a\left(\cdot, t_{n}\right)} \nabla \zeta^{n}\right\| \\
& \quad \leq\left\|\sqrt{a\left(\cdot, t_{n}\right)} \nabla \zeta^{0}\right\|+\lambda \sqrt{\lambda} K \tau_{n}^{\alpha} \Gamma(2-\alpha) \sum_{j=1}^{n} \theta_{n, j}\left\|\nabla \varphi^{j}\right\|+C \sqrt{T^{\alpha} \Gamma(1-\alpha)} h^{2} .
\end{aligned}
$$

By Lemma 3.1, we get $\left\|\varphi^{j}\right\|_{1} \leq C j^{-\min \{2-\alpha, r \alpha\}}$. Substituting this inequality into (25) and recalling (12) yields

$$
\begin{aligned}
\left\|\nabla \zeta^{n}\right\| \leq & \lambda\left\|\nabla \zeta^{0}\right\|+C \lambda^{2} K \tau_{n}^{\alpha} \Gamma(2-\alpha) \sum_{j=1}^{n} \theta_{n, j} j^{-\min \{2-\alpha, r \alpha\}} \\
& +C \sqrt{\lambda} \sqrt{T^{\alpha} \Gamma(1-\alpha)} h^{2} \\
\leq & C \lambda^{2} K T^{\alpha} \Gamma(1-\alpha) N^{-\min \{2-\alpha, r \alpha\}}+C \sqrt{\lambda T^{\alpha} \Gamma(1-\alpha)} h^{2}
\end{aligned}
$$

where we used $\left\|\nabla \zeta^{0}\right\|=\left\|\nabla\left(R_{h} u^{0}-u_{h}^{0}\right)\right\|=0$, then invoked (12) with $\eta=\min \{2-\alpha, r \alpha\}$ for the $j^{-\min \{2-\alpha, r \alpha\}}$ term. Combining this bound and (6) with (18), we get (20).

Let $I_{h}: H^{2}(\Omega) \rightarrow V_{0 h}$ be the associated interpolation operator satisfying $I_{h} u\left(a_{i}\right)=u\left(a_{i}\right)$, where $a_{i},(i=1,2,3,4)$ are the four vertices of $K_{m}$. Imitating the proof given for [35, Lemma 2] yields

$$
\left\|R_{h}(t) w-I_{h} w\right\|_{1} \leq C h^{2}\|u\|_{3}, \quad \forall w \in H_{0}^{1}(\Omega) \cap H^{3}(\Omega)
$$


In order to derive the global superconvergence result, we adopt the same interpolation postprocessing operator $I_{2 h}$ as in [22], which satisfies

$$
\begin{aligned}
& I_{2 h} I_{h} w=I_{2 h} w, \quad \forall w \in H^{2}(\Omega), \\
& \left\|w-I_{2 h} w\right\|_{1} \leq C h^{2}|w|_{3}, \quad \forall w \in H^{3}(\Omega), \\
& \left\|I_{2 h} w_{h}\right\|_{1} \leq C\left\|w_{h}\right\|_{1}, \quad \forall w_{h} \in V_{0 h} .
\end{aligned}
$$

Corollary 4.2 Under the conditions of Theorem 4.1 and assuming $\|u\|_{L^{\infty}\left(H^{3}\right)}$ is finite, let the finite element space be the conforming rectangular bilinear element space, then the following superconvergence estimates hold:

$$
\begin{aligned}
& \left\|I_{h} u^{n}-u_{h}^{n}\right\|_{1} \leq C\left(h^{2}+N^{-\min \{2-\alpha, r \alpha\}}\right), \\
& \left\|u^{n}-I_{2 h} u_{h}^{n}\right\|_{1} \leq C\left(h^{2}+N^{-\min \{2-\alpha, r \alpha\}}\right) .
\end{aligned}
$$

Proof Applying (26) and (21), one has

$$
\begin{aligned}
\left\|I_{h} u^{n}-u_{h}^{n}\right\|_{1} & \leq\left\|I_{h} u^{n}-R_{h}\left(t_{n}\right) u^{n}\right\|_{1}+\left\|R_{h}\left(t_{n}\right) u^{n}-u_{h}^{n}\right\|_{1} \\
& \leq C h^{2}+C\left(h^{2}+N^{-\min \{2-\alpha, r \alpha\}}\right) \\
& \leq C\left(h^{2}+N^{-\min \{2-\alpha, r \alpha\}}\right) .
\end{aligned}
$$

Furthermore, combining this result with (27a)-(27c) yields

$$
\begin{aligned}
\left\|u^{n}-I_{2 h} u_{h}^{n}\right\|_{1} & \leq\left\|u^{n}-I_{2 h} I_{h} u^{n}\right\|_{1}+\left\|I_{2 h} I_{h} u^{n}-I_{2 h} u_{h}^{n}\right\|_{1} \\
& =\left\|u^{n}-I_{2 h} u^{n}\right\|_{1}+\left\|I_{2 h}\left(I_{h} u^{n}-u_{h}^{n}\right)\right\|_{1} \\
& \leq C h^{2}+C\left\|I_{h} u^{n}-u_{h}^{n}\right\|_{1} \\
& \leq C\left(h^{2}+N^{-\min \{2-\alpha, r \alpha\}}\right) .
\end{aligned}
$$

Thus the proof is complete.

\section{Numerical experiments}

We compute numerical solutions for an example of problem (1a)-(1b) that near $t=0$ behaves as described in (4). The $E_{1}^{M, N}$ and $E_{2}^{M, N}$ errors in the computed solutions are defined by

$$
E_{1}^{M, N}:=\max _{0 \leq n \leq N}\left\|I_{h} u^{n}-u_{h}^{n}\right\|_{1}, \quad E_{2}^{M, N}:=\max _{0 \leq n \leq N}\left\|u^{n}-I_{2 h} u_{h}^{n}\right\|_{1} .
$$

Example 5.1 Consider the two-dimensional time-fractional diffusion problem (1a)-(1b) with $\Omega=(0, \pi) \times(0, \pi), a(x, y, t)=t \cos (t) x y / \pi^{2}, T=1$. The function $f$ is chosen such that the exact solution of the problem (1a)-(1b) is $u(x, y, t)=\left(t^{\alpha}+t^{3}\right) \sin x \sin y$.

Corollary 4.2 predicts the rate of convergence $O\left(h^{2}+N^{-\min \{2-\alpha, r \alpha\}}\right)$ for $E_{1}^{M, N}$ and $E_{2}^{M, N}$. We choose a uniform rectangular partition of $\Omega$ with $M+1$ nodes in each spatial direction. Tables 1 and 2 show the $E_{1}^{M, N}$ and $E_{2}^{M, N}$ errors for $\alpha=0.4,0.6,0.8$ with $r=(2-\alpha) / \alpha$. We take 
Table $1 E_{1}^{M, N}$ errors and orders of convergence for L1 FEM with $r=(2-\alpha) / \alpha$

\begin{tabular}{llllll}
\hline & $N=20$ & $N=40$ & $N=80$ & $N=160$ & $N=320$ \\
\hline$\alpha=0.4$ & $8.1943 \mathrm{E}-2$ & $2.9074 \mathrm{E}-2$ & $1.0138 \mathrm{E}-2$ & $3.4975 \mathrm{E}-3$ & $1.2089 \mathrm{E}-3$ \\
& & 1.4949 & 1.5198 & 1.5354 & 1.5325 \\
$\alpha=0.6$ & $8.3329 \mathrm{E}-2$ & $3.1109 \mathrm{E}-2$ & $1.1715 \mathrm{E}-2$ & $4.4376 \mathrm{E}-3$ & $1.6896 \mathrm{E}-3$ \\
& & 1.4214 & 1.4089 & 1.4005 & 1.3931 \\
$\alpha=0.8$ & $1.0196 \mathrm{E}-1$ & $4.1498 \mathrm{E}-2$ & $1.7335 \mathrm{E}-2$ & $7.3639 \mathrm{E}-3$ & $1.1681 \mathrm{E}-3$ \\
& & 1.2968 & 1.2592 & 1.2352 & 1.2168 \\
\hline
\end{tabular}

Table $2 E_{2}^{M, N}$ errors and orders of convergence for L1 FEM with $r=(2-\alpha) / \alpha$

\begin{tabular}{llllll}
\hline & $N=20$ & $N=40$ & $N=80$ & $N=160$ & $N=320$ \\
\hline$\alpha=0.4$ & $8.3912 \mathrm{E}-2$ & $2.9429 \mathrm{E}-2$ & $1.0212 \mathrm{E}-2$ & $3.5165 \mathrm{E}-3$ & $1.2067 \mathrm{E}-3$ \\
& & 1.5116 & 1.6459 & 1.5381 & 1.5391 \\
$\alpha=0.6$ & $8.5231 \mathrm{E}-2$ & $3.1432 \mathrm{E}-2$ & $1.1777 \mathrm{E}-2$ & $4.4528 \mathrm{E}-3$ & $1.6947 \mathrm{E}-3$ \\
& & 1.4391 & 1.4162 & 1.4032 & 1.3969 \\
$\alpha=0.8$ & $1.0362 \mathrm{E}-1$ & $4.1759 \mathrm{E}-2$ & $1.7383 \mathrm{E}-2$ & $7.3766 \mathrm{E}-3$ & $3.1681 \mathrm{E}-3$ \\
& & 1.3111 & 1.2643 & 1.2367 & 1.2193 \\
\hline
\end{tabular}

Table $3 E_{1}^{M, N}$ and $E_{2}^{M, N}$ convergent results on spatial direction for L1 FEM with $\alpha=0.4$

\begin{tabular}{llllll}
\hline & $M=4$ & $M=8$ & $M=16$ & $M=32$ & $M=64$ \\
\hline$E_{1}^{M, N}$ & $4.1198 \mathrm{E}-1$ & $1.0643 \mathrm{E}-1$ & $2.6937 \mathrm{E}-2$ & $6.8276 \mathrm{E}-3$ & $1.7677 \mathrm{E}-3$ \\
& & 1.9526 & 1.9822 & 1.9823 & 1.9476 \\
$E_{2}^{M, N}$ & $5.9157 \mathrm{E}-1$ & $1.4835 \mathrm{E}-1$ & $3.7192 \mathrm{E}-2$ & $9.3590 \mathrm{E}-3$ & $2.3847 \mathrm{E}-3$ \\
& & 1.9958 & 1.9960 & 1.9905 & 1.9725 \\
\hline
\end{tabular}

$M=N$ so that the temporal error dominates the spatial error in the bound of Corollary 4.2. The orders of convergence displayed indicate that the rate of convergence is $N^{-(2-\alpha)}$, as predicted by Corollary 4.2. Table 3 shows the spatial errors and the associated orders of convergence for $\alpha=0.4$ and $r=(2-\alpha) / \alpha$. Here we take $N=2000$ so that the spatial error dominates the results, and we observe $O\left(h^{2}\right)$ convergence, as predicted by Corollary 4.2.

\section{Acknowledgements}

The author wishes to thank the referees and the editor for their valuable comments and suggestions. The author also cordially gives her great gratitude to Dr. Chaobao Huang for this help in numerical experiments.

\section{Funding}

The author is grateful to the National Natural Science Foundation of PR China (Grant Nos. 11801332 and 11971276).

Availability of data and materials

Not applicable.

\section{Competing interests}

The author declares that she has no competing interests.

Author's contributions

Author read and approved the final manuscript.

\section{Publisher's Note}

Springer Nature remains neutral with regard to jurisdictional claims in published maps and institutional affiliations.

Received: 28 April 2020 Accepted: 14 September 2020 Published online: 21 September 2020

\section{References}

1. Ahmad, J., Mohyud-Din, S.T.: An efficient algorithm for some highly nonlinear fractional pdes in mathematical physics. PLOS ONE 9(12), 1-17 (2014) 
2. Alikhanov, A.A.: A new difference scheme for the time fractional diffusion equation. J. Comput. Phys. 280, 424-438 (2015)

3. An, N., Huang, C., Yu, X.: Error analysis of direct discontinuous Galerkin method for two-dimensional fractional diffusion-wave equation. Appl. Math. Comput. 349, 148-157 (2019)

4. An, N., Huang, C., Yu, X.: Error analysis of discontinuous Galerkin method for the time fractional KdV equation with weak singularity solution. Discrete Contin. Dyn. Syst., Ser. B 25(1), 321-334 (2020)

5. Bramble, J.H., Pasciak, J.E., Steinbach, O.: On the stability of the $L^{2}$ projection in $H^{\prime}(\Omega)$. Math. Comput. 71(237), 147-156 (2002)

6. Bu, W., Xiao, A.: An $h-p$ version of the continuous Petrov-Galerkin finite element method for Riemann-Liouville fractional differential equation with novel test basis functions. Numer. Algorithms 81(2), 529-545 (2019)

7. Chen, J., Liu, F., Liu, Q., Chen, X., Anh, V., Turner, I., Burrage, K.: Numerical simulation for the three-dimension fractional sub-diffusion equation. Appl. Math. Model. 38(15-16), 3695-3705 (2014)

8. Gu, Q., Allan Schiff, E., Grebner, S., Wang, F., Schwarz, R.: Non-Gaussian transport measurements and the Einstein relation in amorphous silicon. Phys. Rev. Lett. 76(17), 3196 (1996)

9. Huang, C., An, N., Yu, X.: A fully discrete direct discontinuous Galerkin method for the fractional diffusion-wave equation. Appl. Anal. 97(4), 659-675 (2018)

10. Huang, C., An, N., Yu, X.: A local discontinuous Galerkin method for time-fractional diffusion equation with discontinuous coefficient. Appl. Numer. Math. 151, 367-379 (2020)

11. Huang, C., An, N., Yu, X., Zhang, H.: A direct discontinuous Galerkin method for time-fractional diffusion equation with discontinuous diffusive coefficient. Complex Var. Elliptic Equ. 65(9), 1445-1461 (2019)

12. Huang, C., Stynes, M.: Superconvergence of the direct discontinuous Galerkin method for a time-fractional initial-boundary value problem. Numer. Methods Partial Differ. Equ. 35(6), 2076-2090 (2019)

13. Huang, C., Stynes, M.: Superconvergence of a finite element method for the multi-term time-fractional diffusion problem. J. Sci. Comput. 82(1), Article ID 10 (2020)

14. Huang, C., Stynes, M., An, N.: Optimal $L^{\infty}\left(L^{2}\right)$ error analysis of a direct discontinuous Galerkin method for a time-fractional reaction-diffusion problem. BIT 58(3), 661-690 (2018)

15. Jia, J., Wang, H.: A fast finite volume method for conservative space-time fractional diffusion equations discretized on space-time locally refined meshes. Comput. Math. Appl. 78(5), 1345-1356 (2019)

16. Jin, B., Li, B., Zhou, Z.: Subdiffusion with a time-dependent coefficient: analysis and numerical solution. Math. Comput. 88(319), 2157-2186 (2019)

17. Kassem, M.: FEM for time-fractional diffusion equations, novel optimal error analyses. Math. Comput. 87(313), 2259-2272 (2018)

18. Klammler, F., Kimmich, R.: Geometrical restrictions of incoherent transport of water by diffusion in protein of silica fine particle systems and by flow in a sponge. A study of anomalous properties using an NMR field-gradient technique. Croat. Chem. Acta 65(2), 455-470 (1992)

19. Li, H., Wu, X., Zhang, J.: Numerical solution of the time-fractional sub-diffusion equation on an unbounded domain in two-dimensional space. East Asian J. Appl. Math. 7(3), 439-454 (2017)

20. Li, Z., Yan, Y.: Error estimates of high-order numerical methods for solving time fractional partial differential equations. Fract. Calc. Appl. Anal. 21(3), 746-774 (2018)

21. Liao, H., Li, D., Zhang, J.: Sharp error estimate of the nonuniform $L 1$ formula for linear reaction-subdiffusion equations. SIAM J. Numer. Anal. 56(2), 1112-1133 (2018)

22. Lin, Q., Lin, J.: Finite Element Methods: Accuracy and Improvement, vol. 1. Elsevier, Amsterdam (2007)

23. Lin, Y., Xu, C.: Finite difference/spectral approximations for the time-fractional diffusion equation. J. Comput. Phys. 225(2), 1533-1552 (2007)

24. Luskin, M., Rannacher, R.: On the smoothing property of the Galerkin method for parabolic equations. SIAM J. Numer. Anal. 19(1), 93-113 (1982)

25. Merdan, M., Gökdoğan, A., Yildirim, A., Mohyud-Din, S.T.: Solution of time-fractional generalized Hirota-Satsuma coupled KdV equation by generalised differential transformation method. Int. J. Numer. Methods Heat Fluid Flow 23(5), 927-940 (2013)

26. Mohyud-Din, S., Yildirim, A., Yülüklü, E.: Homotopy analysis method for space-and time-fractional KdV equation. Int. J. Numer. Methods Heat Fluid Flow 22, 928 (2012)

27. Mohyud-Din, S.T., Akram, T., Abbas, M., Ismail, A.I., Ali, N.H.M.: A fully implicit finite difference scheme based on extended cubic B-splines for time fractional advection-diffusion equation. Adv. Differ. Equ. 109, 17 (2018)

28. Mohyud-Din, S.T., Bibi, S., Ahmed, N., Khan, U.: Some exact solutions of the nonlinear space-time fractional differential equations. Waves Random Complex Media 29(4), 645-664 (2019)

29. Mohyud-Din, S.T., Jabeen Awan, F., Ahmad, J., Hassan, S.M.: Differential transform method with complex transforms to some nonlinear fractional problems in mathematical physics. Math. Probl. Eng. 9, Article ID 364853 (2015)

30. Mohyuddin, S.T., Asad lqbal, M., Hassan, S.M.: Modified Legendre wavelets technique for fractional oscillation equations. Entropy 17(10), 6925-6936 (2015)

31. Mustapha, K., Abdallah, B., Furati, K.M.: A discontinuous Petrov-Galerkin method for time-fractional diffusion equations. SIAM J. Numer. Anal. 52(5), 2512-2529 (2014)

32. Natalia, K.: Error analysis of the $L 1$ method on graded and uniform meshes for a fractional-derivative problem in two and three dimensions. Math. Comput. 88(319), 2135-2155 (2019)

33. Porto, M., Bunde, A., Havlin, S., Roman, H.E.: Structural and dynamical properties of the percolation backbone in two and three dimensions. Phys. Rev. E 56(2), 1667 (1997)

34. Ren, J., Huang, C., An, N.: Direct discontinuous Galerkin method for solving nonlinear time fractional diffusion equation with weak singularity solution. Appl. Math. Lett. 102, 106111 (2020)

35. Shi, D.Y., Wang, F.L., Fan, M.Z., Zhao, Y.M.: A new approach of the lowest-order anisotropic mixed finite element high-accuracy analysis for nonlinear sine-Gordon equations. Math. Numer. Sin. 37(2), 148-161 (2015)

36. Stynes, M., O'Riordan, E., Gracia, J.L.: Error analysis of a finite difference method on graded meshes for a time-fractional diffusion equation. SIAM J. Numer. Anal. 55(2), 1057-1079 (2017)

37. Thomée, V.: Galerkin Finite Element Methods for Parabolic Problems. 2nd Revised and Expanded Edition. Springer Berlin (2006) 
38. Vong, S., Lyu, P.: On numerical contour integral method for fractional diffusion equations with variable coefficients. Appl. Math. Lett. 64, 137-142 (2017)

39. Wang, F., Chen, H., Wang, H.: Finite element simulation and efficient algorithm for fractional Cahn-Hilliard equation. J. Comput. Appl. Math. 356, 248-266 (2019)

40. Weber, H.W., Kimmich, R.: Anomalous segment diffusion in polymers and NMR relaxation spectroscopy. Macromolecules 26(10), 2597-2606 (1993)

41. Yang, S., Chen, H., Wang, H.: Least-squared mixed variational formulation based on space decomposition for a kind of variable-coefficient fractional diffusion problems. J. Sci. Comput. 78(2), 687-709 (2019)

42. Yin, B., Liu, Y., Li, H., Zhang, Z:: Finite element methods based on two families of second-order numerical formulas for the fractional cable model with smooth solutions. J. Sci. Comput. 84(1), 2 (2020)

43. Yuan, Q., Chen, H.: An expanded mixed finite element simulation for two-sided time-dependent fractional diffusion problem. Adv. Differ. Equ. 34, 15 (2018)

44. Zeng, F., Li, C., Liu, F., Turner, I.: The use of finite difference/element approaches for solving the time-fractional subdiffusion equation. SIAM J. Sci. Comput. 35(6), a2976-a3000 (2013)

45. Zhang, H., Shi, D.: Superconvergence analysis for time-fractional diffusion equations with nonconforming mixed finite element method. J. Comput. Math. 37(4), 527-544 (2019)

46. Zhao, J., Li, H., Fang, Z., Liu, Y.: A mixed finite volume element method for time-fractional reaction-diffusion equations on triangular grids. Mathematics 7, 600 (2019)

47. Zhao, Y., Zhang, Y., Liu, F., Turner, I., Tang, Y., Anh, V.: Convergence and superconvergence of a fully-discrete scheme for multi-term time fractional diffusion equations. Comput. Math. Appl. 73(6), 1087-1099 (2017)

48. Zhou, Z., Tan, Z.: Finite element approximation of optimal control problem governed by space fractional equation. J. Sci. Comput. 78(3), 1840-1861 (2019)

\section{Submit your manuscript to a SpringerOpen ${ }^{\circ}$ journal and benefit from:}

- Convenient online submission

- Rigorous peer review

- Open access: articles freely available online

- High visibility within the field

- Retaining the copyright to your article

Submit your next manuscript at $\gg$ springeropen.com 